\title{
Trasplante cardiaco y bloqueo auriculoventricular tardío. Reporte de un caso
}

\author{
Heart transplant and delayed atrioventricular block. Case report
}

Alberto Navarro-Navajas', José Cruz-Cuevas², Adrián Bolívar Mejía ${ }^{*}$ y Daniel Isaza ${ }^{4}$

${ }^{1}$ Servicio de Cardiología, Fundación Cardioinfantil Instituto de Cardiología, y Posgrado en Cardiología, Universidad El Bosque, Bogotá; ${ }^{2}$ Servicio de Medicina Interna, Fundación Cardioinfantil, Instituto de Cardiología, y Posgrado de Medicina Interna, Universidad del Rosario, Bogotá; ${ }^{3}$ Servicio de Medicina Interna, Clínica Cardio VID, y Posgrado de Cardiología, Universidad Pontificia Bolivariana, Medellín; ${ }^{4}$ Dirección de Unidad de Cuidado Coronario, Fundación Cardioinfantil, Instituto de Cardiología, Bogotá. Colombia

\section{Introducción}

Si bien se ha avanzado de manera exponencial en el tratamiento de la falla cardiaca, el $1-10 \%$ de los pacientes progresa a falla cardiaca avanzada, síndrome caracterizado por deterioro clínico continuo, caquexia, intolerancia a la titulación de medicación, resistencia a diuréticos y episodios frecuentes de descompensación; estadio en el cual el trasplante cardiaco ortotópico es el manejo de elección. Sin embargo, el corazón trasplantado carece de inervación y regulación autonómica, y esto, asociado al trauma quirúrgico y al rechazo, la disfunción o la vasculopatía del injerto, condiciona un importante riesgo de arritmias cardiacas y muerte súbita.

\section{Caso clínico}

Mujer de 50 años con antecedente de trasplante cardiaco ortotópico 7 años atrás debido a falla cardiaca avanzada de etiología chagásica. Historia de rechazo celular moderado y angina crónica por vasculopatía grave del injerto no revascularizable. Recibía tratamiento con ácido acetilsalicílico, carvedilol, ivabradina, everolimus y tacrolimus a dosis terapéuticas. Se encontraba en clase funcional II/IV de la New York Heart Association. Consultó a urgencias por 5 días de deterioro de su clase funcional y edema de miembros inferiores, sin otra sintomatología asociada.

En la exploración física presentó presión arterial de $117 / 83 \mathrm{mmHg}$, bradicardia (42 latidos por minuto) y edema de grado 2 de miembros inferiores. Se documentaron hemograma, electrolitos, función renal y TSH normales. El péptido natriurético cerebral fue de 1028,90 pg/ml. Las concentraciones de tacrolimus y everolimus estaban en metas. El electrocardiograma mostró bradicardia sinusal, bloqueo completo de rama derecha y bloqueo auriculoventricular de segundo grado Mobitz II (Fig. 1). El ecocardiograma transtorácico evidenció una fracción de eyección del ventrículo izquierdo del $40 \%$.

Teniendo en cuenta el bloqueo auriculoventricular documentado y la bradicardia asociada, así como el antecedente de rechazo celular, vasculopatía y disfunción del injerto, se consideró candidata a cardiodesfibrilador implantable (CDI), el cual se implantó sin complicaciones.

\section{Discusión}

Dentro del espectro clínico de la falla cardiaca, el estadio D contempla el trasplante cardiaco ortotópico como la mejor opción terapéutica. La evolución de las técnicas quirúrgicas y la mejora en el tratamiento inmunosupresor 


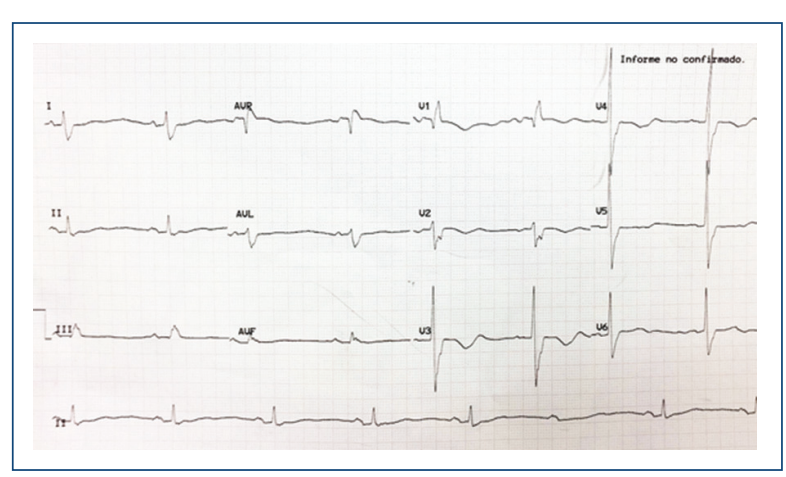

Figura 1. Electrocardiograma con boqueo completo de rama derecha del haz de His asociado a bloqueo auriculoventricular de segundo grado Mobitz II.

han logrado impactar positivamente en el pronóstico a largo plazo tras un trasplante cardiaco, estimándose una sobrevida del $90 \%$ a 5 años y del $70 \%$ a 10 años ${ }^{1}$. Este aumento en la sobrevida, así como diversos cambios fisiopatológicos asociados al estado postrasplante, han llevado a una mayor aparición de complicaciones a largo plazo, entre ellas las bradiarritmias.

Se han descrito los mecanismos y los sustratos fisiopatológicos asociados a las arritmias postrasplante ${ }^{2,3}$, incluyendo el tiempo de isquemia del injerto, el compromiso del sistema cardionector en relación con el tipo de técnica quirúrgica, la denervación y la potencial reinervación irregular del injerto, el rechazo, la vasculopatía y la disfunción del injerto, y los efectos adversos asociados al uso de medicamentos con efecto cronotrópico negativo².

El caso presentado corresponde a una bradiarritmia postrasplante asociada a bloqueo auriculoventricular de segundo grado Mobitz II de presentación tardía. Si bien dicha alteración fue documentada en el contexto del uso de carvedilol e ivabradina, se debe tener en cuenta que a las dosis instauradas de dichos fármacos no se considera que estos sean la causa del bloqueo auriculoventricular, más aún teniendo en cuenta el mecanismo de acción de la ivabradina en el nodo sinusal ${ }^{3,4}$. Adicionalmente, dichos medicamentos se venían empleando como parte de la terapia médica requerida por la paciente, encontrándose entonces indicado el implante de un dispositivo de estimulación eléctrica para el tratamiento de las bradiarritmias asociadas a su uso ${ }^{4}$.

Según los reportes en la literatura, el 8-30\% de los pacientes con trasplante cardiaco ortotópico presentan alguna bradiarritmia, siendo más frecuente la disfunción sinusal, seguida de las alteraciones en la conducción auriculoventricular ${ }^{3,4}$, las cuales se presentan de forma más tardía dado que se relacionan con el envejecimiento del sistema de conducción o, hasta en el 30\% de los casos, con vasculopatía del injerto ${ }^{3}$.

El implante de un CDI en pacientes receptores de trasplante cardiaco es controvertido debido a que su efectividad no ha sido demostrada de manera concordante. Según las guías vigentes para la prevención de la muerte súbita cardiaca, es razonable considerar el implante de un $\mathrm{CDI}$ en aquellos pacientes que tengan disfunción ventricular izquierda, vasculopatía del injerto grave y una expectativa de vida mayor de 1 año (recomendación Ilb, nivel de evidencia B-NR) ${ }^{5}$.

Aun cuando en estudios previos se ha reportado con mayor proporción asistolia 0 actividad eléctrica sin pulso asociada a la muerte súbita en pacientes postrasplante cardiaco (34 y 20\%, respectivamente), la fibrilación ventricular puede identificarse en el 6-10\% de los casos, encontrándose como factores de riesgo asociados a su presentación el antecedente de rechazo, vasculopatía y disfunción del injerto, hallazgos que en conjunto se han relacionado con una mayor incidencia de descargas apropiadas en pacientes con trasplante cardiaco llevados a implante de CDI, y que condicionan un riesgo de muerte súbita que ha sido considerado comparable al de los pacientes con falla cardiaca con fracción de eyección reducida, lo cual en nuestro caso motivó el implante de un CDI para prevención primaria de muerte súbita ${ }^{6,7}$.

\section{Conclusión}

Las bradiarritmias son frecuentes en los pacientes llevados a trasplante cardiaco. La principal alteración asociada es la disfunción sinusal; la presencia de bloqueos auriculoventriculares es menos frecuente. Estos últimos suelen presentarse tardíamente y asociados a vasculopatía del injerto. El tratamiento de elección es el implante de un marcapasos, el cual tiene como objetivo disminuir los síntomas asociados y el riesgo de muerte súbita. Aun cuando la utilidad del CDI en los pacientes receptores de trasplante cardiaco permanece incierta, es razonable considerar su uso en aquellos pacientes con antecedente de vasculopatía, rechazo y disfunción del injerto.

\section{Conflictos de intereses}

Los autores declaran no tener ningún conflicto de intereses. 


\section{Financiación}

La presente investigación no ha recibido ninguna beca específica de agencias de los sectores público, comercial, o sin ánimo de lucro.

\section{Responsabilidades éticas}

Protección de personas y animales. Los autores declaran que para esta investigación no se han realizado experimentos en seres humanos ni en animales.

Confidencialidad de los datos. Los autores declaran que han seguido los protocolos de su centro de trabajo sobre la publicación de datos de pacientes.

Derecho a la privacidad y consentimiento informado. Los autores declaran que en este artículo no aparecen datos de pacientes.

\section{Bibliografía}

1. Ross M, Kouretas P, Gamberg P, Miller J, Burge M, Reitz B, et al. Tenand 20 -year survivors of pediatric orthotopic heart transplantation. J Heart Lung Transplant. 2006;25:261-70.

2. Thajudeen A, Stecker C, Shehata M, Patel J, Wang X, McAnulty J. Arrhythmias after heart transplantation: mechanisms and management. $J$ Am Heart Assoc. 2012;1:e001461.

3. Hamon, D, Taleski J, Vaseghi M, Shivkumar K, Boyle NG. Arrhythmias in the heart transplant patient. Arrhythm Electrophysiol Rev. 2014;3:149-55.

4. Kusumoto FM, Schoenfeld MH, Barrett C, Edgerton JR, Ellenbogen KA, Gold MR, et al. 2018 ACC/AHA/HRS Guideline on the Evaluation and Management of Patients With Bradycardia and Cardiac Conduction Delay: Executive Summary: A Report of the American College of Cardiology/American Heart Association Task Force on Clinical Practice Guidelines, and the Heart Rhythm Society. J Am Coll Cardiol. 2019;74:932-87.

5. Al-Khatib SM, Stevenson WG, Ackerman MJ, Bryant WJ, Callans DJ, Curtis AB, et al. $2017 \mathrm{AHA} / \mathrm{ACC} / \mathrm{HRS}$ guideline for management of patients with ventricular arrhythmias and the prevention of sudden cardiac death: a report of the American College of Cardiology/American Heart Association Task Force on Clinical Practice Guidelines and the Heart Rhythm Society. Heart Rhythm. 2018;15:e73-e189.

6. Alba AC, Fan CS, Manlhiot C, Dipchand Al, Stehlik J, Ross HJ. The evolving risk of sudden cardiac death after heart transplant. An analysis of the ISHLT Thoracic Transplant Registry. Clin Transplant. 2019; 33:e13490.

7. Eskander MA, Adler E, Hoffmayer KS. Arrhythmias and sudden cardiac death in post-cardiac transplant patients. Curr Opin Cardiol. 2020;35:308-11. 